\title{
DETECÇÃO DE RECIRCULAÇÃO INTERNA DE GASES ATRAVÉS DE TESTES EM PLANTA DE DESGASEIFICAÇÃO A VÁCUO *
}

Volney Viana Nogueira'

\section{Resumo}

A recirculação interna de gases em bombas de vácuo é uma falha frequente em estações de desgaseificação de aço e está associada ao aumento dos tempos de processo, a um maior consumo energético e a não obtenção da pressão de trabalho adequada. O tratamento de tais desvios pode ser complicado pela falta de informação disponível sobre os modos de falha dos equipamentos. Este artigo descreve um método experimental para encontrar valores de referência para testes que podem ser realizados na planta para a detecção de não conformidades em bombas de vácuo constituídas de ejetores a vapor e condensadores; tal método é composto por testes lógicos, sequenciais e não intrusivos. São fornecidas tabelas com os valores esperados das grandezas testadas, assim como diversas causas de redução de desempenho e de perda de função dos componentes.

Palavras-chave: Desgaseificação a vácuo; Passagem interna em válvulas; Recirculação de gases; Infiltração.

\section{DETECTION OF INTERNAL GAS RECIRCULATION THROUGH TESTS ON A VACUUM DEGASSING PLANT}

\section{Abstract}

Internal gas recirculation in vacuum pumps is a frequent failure in steel degassing stations and is associated with increased process times, higher energy consumption and failure to obtain adequate working pressure. The treatment of such deviations can be complicated by the lack of available information on the failure modes of the equipment. This article describes an experimental method to find reference values for tests that can be performed in the plant for the detection of non-conformities in vacuum pumps consist of ejectors steam and condensers; the method is composed of logical, sequential and non-intrusive tests. Tables are provided with the expected values of the tested quantities, as well as several causes of reduced performance and loss of function of the components.

Keywords: Vacuum degasser; passage valves; Gas recirculation; Leakage.

Engenheiro Mecânico, Graduado na Universidade Federal de Uberlândia, Mestrando em Engenharia Química na Universidade Federal de São João Del Rei, Assessor Técnico de Manutenção na Gerdau, Ouro Branco, Minas Gerais, Brasil. 


\section{INTRODUÇÃO}

Uma planta de desgaseificação tem como função principal retirar gases que estão diluídos no aço, a partir da produção de vácuo dentro de um vaso onde o metal líquido é armazenado. Conforme Oliva [1], as tecnologias tradicionais utilizadas na geração de vácuo para o tratamento de aços são ejetores a vapor, bombas de vácuo de anel líquido e sistemas combinados entre esses dois.

Uma bomba de vácuo compreende todos os equipamentos e componentes entre 0 ejetor 1 e a chaminé e possui pressões de vácuo diferentes em diferentes pontos do conjunto. Na figura 1 e na tabela I é possível verificar que a pressão reduz em direção ao ejetor 1 . 0 degradê das cores da figura explicita os diferentes estágios de pressão.

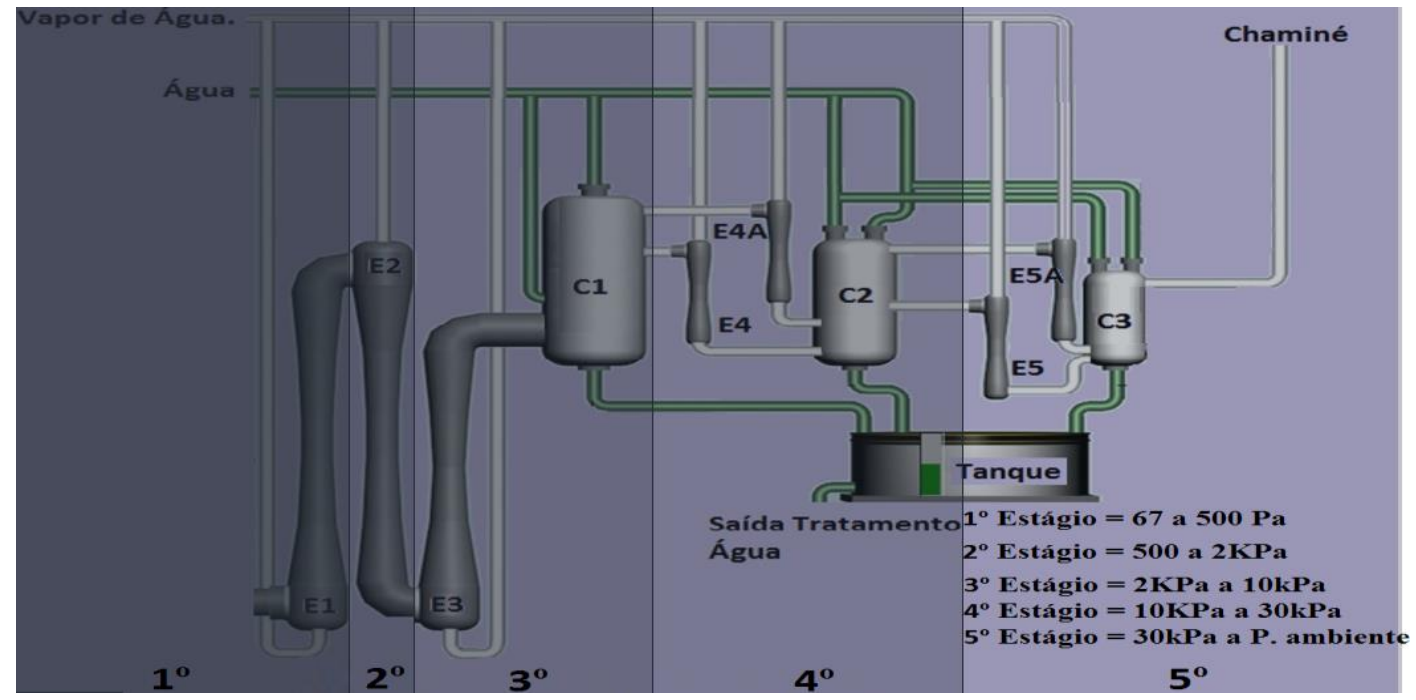

Figura 1: Estágios da Bomba de Vácuo.

O bocal impelente, indicado na figura 2, é dimensionado para que a velocidade do vapor seja supersônica, conforme Ren, L.Q. et al. 2014 e Nozzle Applet [2]. O vapor chega à câmara do bocal em velocidade subsônica, logo no início do bocal impelente atinge a velocidade sônica, e à sua saída, chega à velocidade supersônica, atingindo entre $900 \mathrm{~m} / \mathrm{s}$ e $1200 \mathrm{~m} / \mathrm{s}$, conforme El-Dessoouky [3]. Ele também é projetado para ter a máxima pressão de descarga de vapor, conforme Martin[4]. 

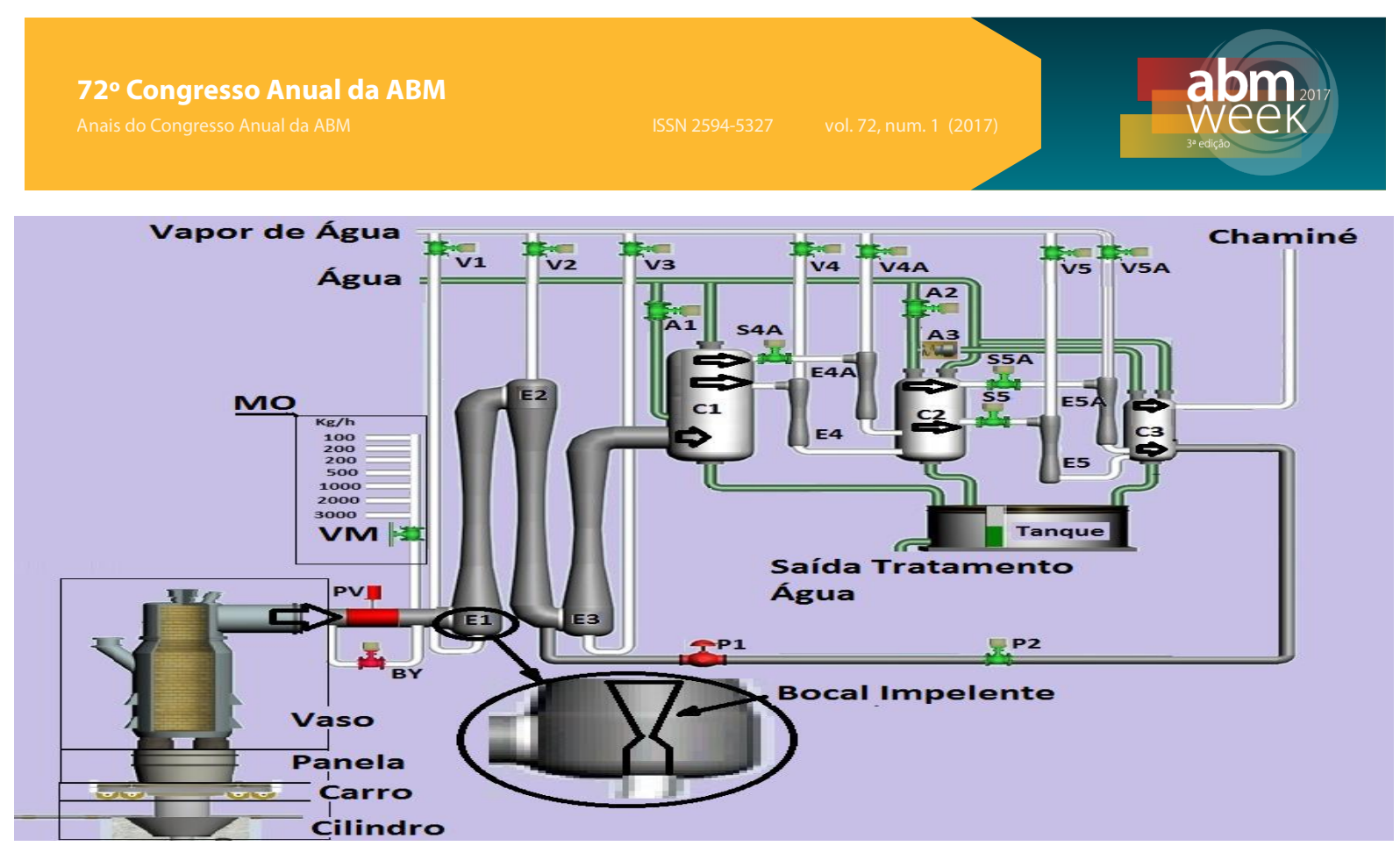

Figura 2: Bomba de vácuo - representação esquemática com válvulas.

Para simplificar a descrição, serão utilizadas várias abreviações de válvulas, ejetores e condensadores conforme tabela I.

Tabela I: Abreviações utilizadas para equipamentos e componentes.

\begin{tabular}{|c|c|c|c|}
\hline Descrição & Sigla & Descrição & Sigla \\
\hline Condensador 1 & C1 & Ejetor 3 & E3 \\
\hline Condensador 2 & $\mathrm{C} 2$ & Ejetor 4 & E4 \\
\hline Condensador 3 & C3 & Ejetor 5 & E5 \\
\hline Ejetor 1 & E1 & Ejetor $5^{\mathrm{a}}$ & E5A \\
\hline Ejetor 2 & E2 & Manifold & $\mathrm{MO}$ \\
\hline $\begin{array}{l}\text { Válvula alimentação água } \\
\text { para C1 }\end{array}$ & A1 & $\begin{array}{l}\text { Válvula linha sucção anterior } \\
\text { E5A }\end{array}$ & S5A \\
\hline $\begin{array}{l}\text { Válvula alimentação água } \\
\text { C2/C3 }\end{array}$ & A2 & $\begin{array}{l}\text { Válvula liberação vapor para } \\
\text { E1 }\end{array}$ & V1 \\
\hline $\begin{array}{l}\text { Válvula de retenção da linha } \\
\text { de } \\
\text { Alimentação de áqua para C3 }\end{array}$ & A3 & $\begin{array}{l}\text { Válvula de liberação de Vapor } \\
\text { para o E2 }\end{array}$ & V2 \\
\hline $\begin{array}{lcc}\text { Válvula by pass linha } \\
\text { principal vácuo }\end{array}$ & BY & Válvula liberação vapor p/ E3 & V3 \\
\hline $\begin{array}{l}\text { Válvula controladora de } \\
\text { vácuo }\end{array}$ & P1 & $\begin{array}{l}\text { Válvula liberação vapor para } \\
\text { E4 }\end{array}$ & V4 \\
\hline Válvula linha Controle vácuo & $\mathrm{P} 2$ & Válvula liberação vapor p/ E4A & V4A \\
\hline Válvula principal de vácuo & PV & $\begin{array}{l}\text { Válvula liberação vapor para } \\
\text { E5 }\end{array}$ & V5 \\
\hline $\begin{array}{l}\text { Válvula linha sucção anterior } \\
\text { E4A. }\end{array}$ & S4A & Válvula liberação vapor p/ E5A & V5A \\
\hline $\begin{array}{l}\text { Válvula linha sucção anterior } \\
\text { a E5 }\end{array}$ & S5 & Válvula manual entrada $M O$ & VM \\
\hline
\end{tabular}

\subsection{VÁLVULAS A3, S4A, S5A e P2}

As válvulas $\mathrm{A} 3, \mathrm{P} 2$, S4A e S5A, estanques, não devem permitir a recirculação de gás dentro da bomba de vácuo. A3 é uma válvula de retenção, não possui acionamento, S4A, S5A e P2 possuem acionamento. A função da válvula P1 na 
tubulação entre C3 e entrada de E3 é permitir uma passagem de gás no sentindo de E3, mantendo uma pressão de vácuo de $30 \mathrm{kPa}$ ou $300 \mathrm{mbar}$, conhecida como prévácuo, antes de iniciar o tratamento de corridas. Ao iniciar o tratamento, a válvula de controle P1 é fechada e P2 com função estanque é fechada também.

\subsection{MANIFOLD COM ORIFÍCIOS CALIBRADOS}

A bomba de vácuo possui um equipamento chamado de Manifold com orifícios Calibrados (MO), representado na figura 2 e destacado na figura 3, que está acoplado entre a entrada de E1 e a válvula principal de vácuo. Ele possui orifícios calibrados que permitem a entrada de ar de forma controlada, simulando os gases succionados do aço durante o tratamento; além disso, simula todos os gases injetados nas pernas e possíveis infiltrações de ar externo que todo o sistema pode ter. Quando é necessário fazer algum teste na planta, a PV é fechada e a válvula manual VM na entrada do manifold é aberta.

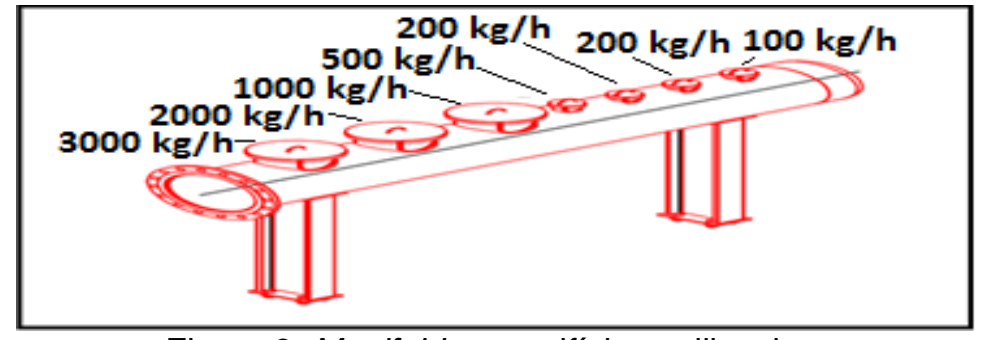

Figura 3: Manifold com orifícios calibrados.

\section{MATERIAIS E MÉTODOS}

A bomba de vácuo da instalação ensaiada possui a capacidade nominal de sucção equivalente a $800 \mathrm{~kg} / \mathrm{h}$ de ar atmosférico a uma pressão de $67 \mathrm{~Pa}$ ou 0,67 mbar. Esta condição simula uma panela de aço em tratamento com sucção de todos os gases do metal líquido, acrescida de possíveis infiltrações externas e demais gases que são injetados no sistema e succionados pela bomba de vácuo.

Os experimentos simularão: a condição real de aço em tratamento, com $800 \mathrm{~kg} / \mathrm{h}$ de ar, uma condição mais favorável com $0 \mathrm{~kg} / \mathrm{h}$ de ar (infiltração interna ou recirculação tão grande que, somada a $800 \mathrm{~kg} / \mathrm{h}$ de ar, a bomba de vácuo ultrapasse seu limite de sucção, por isso $0 \mathrm{~kg} / \mathrm{h}$ ) e uma condição mais desfavorável com $2.000 \mathrm{~kg} / \mathrm{h}$ de ar (uma infiltração interna ou recirculação tão pequena, que na condição de projeto, $800 \mathrm{~kg} / \mathrm{h}$, a bomba absorva, e os testes não detectem alteração).

Para a realização dos testes é preciso que os parâmetros de vapor e água estejam dentro da normalidade: vapor a temperatura entre $463 \mathrm{~K}$ e $503 \mathrm{~K} \quad\left(190^{\circ} \mathrm{C}\right.$ e $\left.230^{\circ} \mathrm{C}\right)$ e pressão entre 1 e 1,05 MPa (10 e 10,5 bar); água a temperatura máxima $307 \mathrm{~K}$ (34 $\left.{ }^{\circ} \mathrm{C}\right)$. Uma inspeção na planta para detecção de infiltração externa e um teste de capacidade de sucção dos ejetores devem ser realizados antes dos experimentos.

A recirculação é a causa principal da impossibilidade de baixar o vácuo; na planta, ela só existe se houver passagem interna em quatro válvulas: A3, S4A, S5A e P2. Serão encontrados valores de referência do sistema sem falha e com falha total, simulando não-conformidade destas válvulas.

As linhas azuis nas próximas figuras representam os fluxos normais do ar na bomba de vácuo, as linhas em vermelho representam os fluxos de ar que não deveriam existir, causando recirculação interna. As representações de válvulas em verde, indicam válvulas abertas e em vermelho, válvulas fechadas. 


\section{EXPERIMENTAÇÃO, RESULTADOS E DISCUSSÃO}

Os experimentos a seguir consideram que a planta está em perfeito funcionamento, porque só assim as referências poderão ser encontradas. Para o desenvolvimento dos experimentos, a abertura das válvulas da linha sucção deve ser realizada antes da abertura das válvulas de liberação de vapor. A válvula $A 1$, que libera passagem de água para o C1 (vide figura 2) deve ficar fechada durante todos os experimentos, pois não receberá carga dos ejetores E1, E2 e E3. Serão detalhados 4 experimentos, um para cada uma das 4 válvulas, A3, S4A, S5A e P2. Para cada válvula, um determinado tempo e pressão de estabilidade deverá ser encontrado devido à posição da mesma no sistema.

\subsection{VÁLVULA SHUT OFF P2 DE CONTROLE DE VÁCUO}

Abrem-se as válvulas das linhas de sucção e de passagem de vapor e gás S4A, S5 e S5A (figura 4), que são de passagem de vapor e ar proveniente do manifold $M O$, e também as válvulas de liberação de vapor V4, V5A, V5 e V5A, todas através do supervisório; dessa forma, os quatros ejetores E4, E4A, E5 e E5A entram em funcionamento.

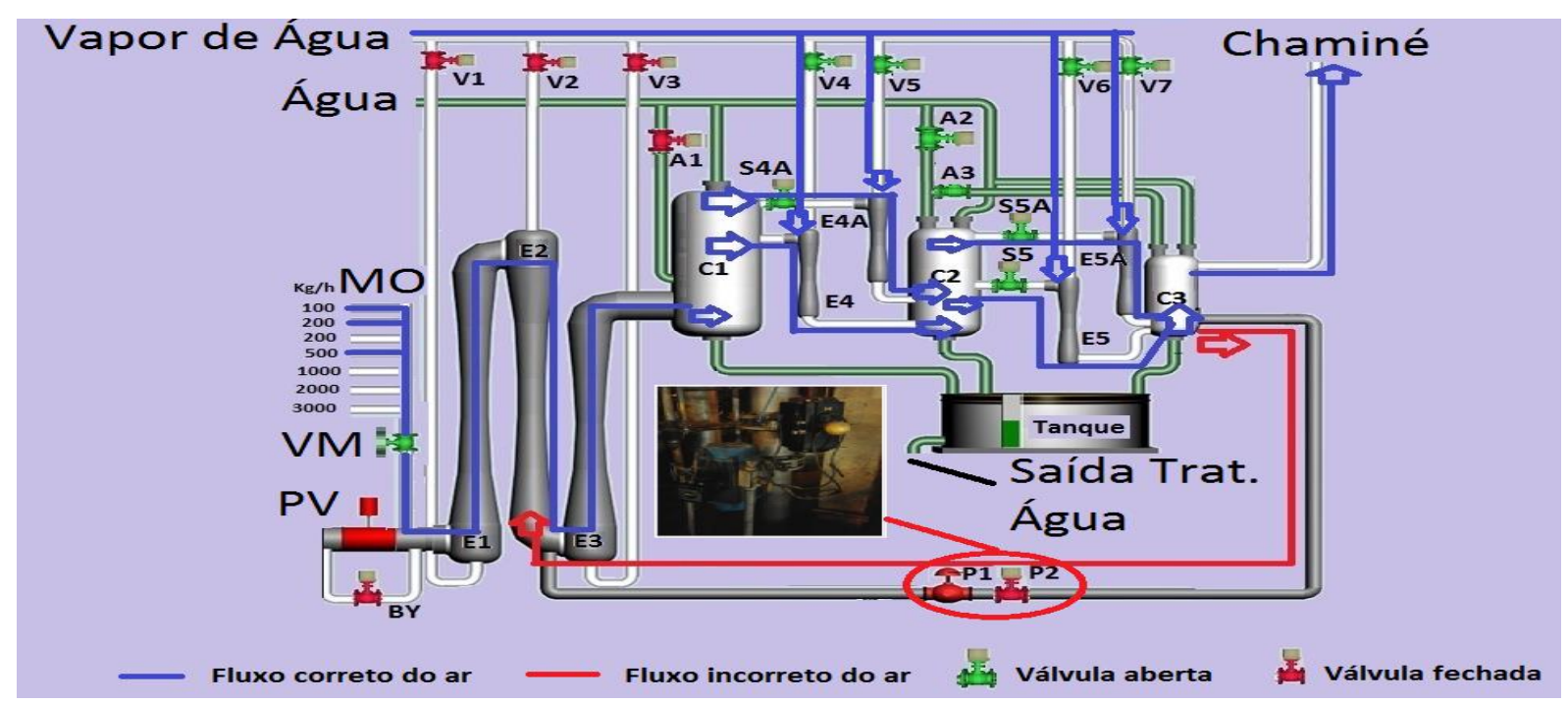

Figura 4: Recirculação através de P1 e P2.

Abre-se a válvula $\mathrm{A} 2$ de passagem de água via supervisório. Dessa forma, já se elimina a possibilidade de passagem interna na válvula $A 3$, de $C 3$ para $C 2$, pois a mesma permanecerá aberta com passagem plena de água. A válvula S4A aberta com E4A aberta, forçando vapor para baixo, garante não existir passagem interna de $\mathrm{C} 2$ para $\mathrm{C} 1$ através de S4A. A válvula S5A aberta com E5A aberta, forçando vapor para baixo, garante não existir passagem interna de C3 para C1 através de S5A. Só poderá existir a falha, passagem interna, se uma válvula permitir passagem de fluxo no momento que ela está fechada. A passagem interna em A3, S4A e S5A será detalhada nos experimentos a frente.

No manifold com orifícios calibrados, é deixada a válvula manual VM de entrada fechada e verificada a condição de estabilidade do sistema, ou seja, a pressão de 
vácuo do sistema cai e se estabiliza. Conforme teste real, a pressão atinge $4 \mathrm{kPa}$ em cerca de 4 minutos. Encerrado o vácuo, desligam-se todos os ejetores e inunda-se a bomba de vácuo com ar.

Repete-se o experimento com a VM de entrada do manifold aberta e as tampas dos orifícios calibrados abertas, permitindo uma entrada de ar de $800 \mathrm{~kg} / \mathrm{h}$. Como esperado, a pressão de vácuo se estabiliza em um patamar maior, em 4,99 kPa. Novamente, é encerrado o vácuo, desligados todos os ejetores e inunda-se a bomba de vácuo com ar. Com $2.000 \mathrm{~kg} / \mathrm{h}$ de entrada de ar, a pressão de vácuo se estabiliza em $5,75 \mathrm{kPa}$ (57,5 mbar). A variação na pressão máxima foi de $\pm 0,03 \mathrm{kPa}(0,3$ mbar).

Conhecendo-se os valores anteriores, que são agora referências, com a bomba de vácuo em funcionamento normal, sem falhas, é possível realizar testes e comparar os resultados. Se a pressão de vácuo for maior, há uma passagem na Válvula shut off de vácuo P2 e válvula de controle de vácuo P1. Como P1 não é estanque, podese considerar passagem interna em P2, que deve ser estanque.

Caso a válvula P2 esteja em falha, com passagem interna plena, devem-se conhecer os respectivos valores de pressão de vácuo. Para tanto, será realizado um novo experimento, simulando passagem plena em P2. É necessário que a válvula P1 seja aberta, utilizando uma permissão de acesso via PLC, para não influenciar o teste de P2. Após aberta P1, abre-se P2 via supervisório, criando uma passagem livre de C3 para entrada de E3. Repetidos os procedimentos dos experimentos já citados, é encontrado o valor de $35 \mathrm{kPa}$ (350 mbar) com a válvula VM fechada, e de $39,4 \mathrm{kPa}$ (394 mbar) para uma entrada de ar de $800 \mathrm{~kg} / \mathrm{h}$ e de 44,1 kPa (441 mbar) com $2.000 \mathrm{~kg} / \mathrm{ar}$ de entrada de ar através do manifold; a variação máxima foi de \pm $0,09 \mathrm{kPa}(0,9 \mathrm{mbar})$. Obtém-se então os valores de referência constantes na tabela II.

Tabela II: Valores de pressão do sistema com e sem falha em P2

\begin{tabular}{|c|c|c|}
\hline $\begin{array}{c}\text { Vazão de Ar } \\
\text { pelo Manifold }\end{array}$ & $\begin{array}{c}\text { Pressão de Vácuo - } \\
\text { sem passagem interna } \\
\text { em P2 }\end{array}$ & $\begin{array}{c}\text { Pressão de Vácuo - com } \\
\text { passagem interna plena em P2 }\end{array}$ \\
\hline $0 \mathrm{~kg} / \mathrm{h}$ & $4,00 \mathrm{kPa}(40 \mathrm{mbar})$ & $35,0 \mathrm{kPa}(350 \mathrm{mbar})$ \\
\hline $800 \mathrm{~kg} / \mathrm{h}$ & $4,99 \mathrm{kPa}(49,9 \mathrm{mbar})$ & $39,4 \mathrm{kPa}(394 \mathrm{mbar})$ \\
\hline $2000 \mathrm{~kg} / \mathrm{h}$ & $5,75 \mathrm{kPa}(57,5 \mathrm{mbar})$ & $44,1 \mathrm{kPa}(441 \mathrm{mbar})$ \\
\hline
\end{tabular}

Valores entre a segunda e a terceira coluna indicam falha na válvula P2. Sabe-se que, em caso de perda da função, os valores são próximos aos da terceira coluna. Valores mais próximos da segunda coluna indicam sujeira ou desgaste na sede da válvula. Oportunidade de melhoria: a planta não possui uma válvula manual na linha entre C3 e entrada de E3, mas se esta válvula for instalada, ela poderá ser fechada assim que o início do tratamento for iniciado, para ter a comprovação que P2 ou P1 e P2 estão com falha.

\subsection{VÁLVULA SHUT OFF DE PASSAGEM GÁS S4A}

Durante o tratamento de corridas, V4A e S4A são abertas apenas no início do processo, trabalhando como um motor de arranque, auxiliando na partida junto com V4. Por essa razão, a linha de sucção entre $C 1$ e $C 2$, passando por E4A, é paralela à linha de sucção entre $\mathrm{C} 1$ e $\mathrm{C} 2$, passando por E4. Perceba que não existe uma válvula shut off na linha de sucção entre C1 e E4 porque sempre existe a passagem de gás e vapor nesta linha sentido C2. 
Quando o ejetor E3 entra em funcionamento, V4A e S4A são fechadas. S4A tem a função de estancar uma recirculação de gás, pois a pressão de vácuo em C1 é menor que em $\mathrm{C} 2$ durante o funcionamento da bomba de vácuo. Os procedimentos citados a seguir podem ser acompanhados através da figura 5 .

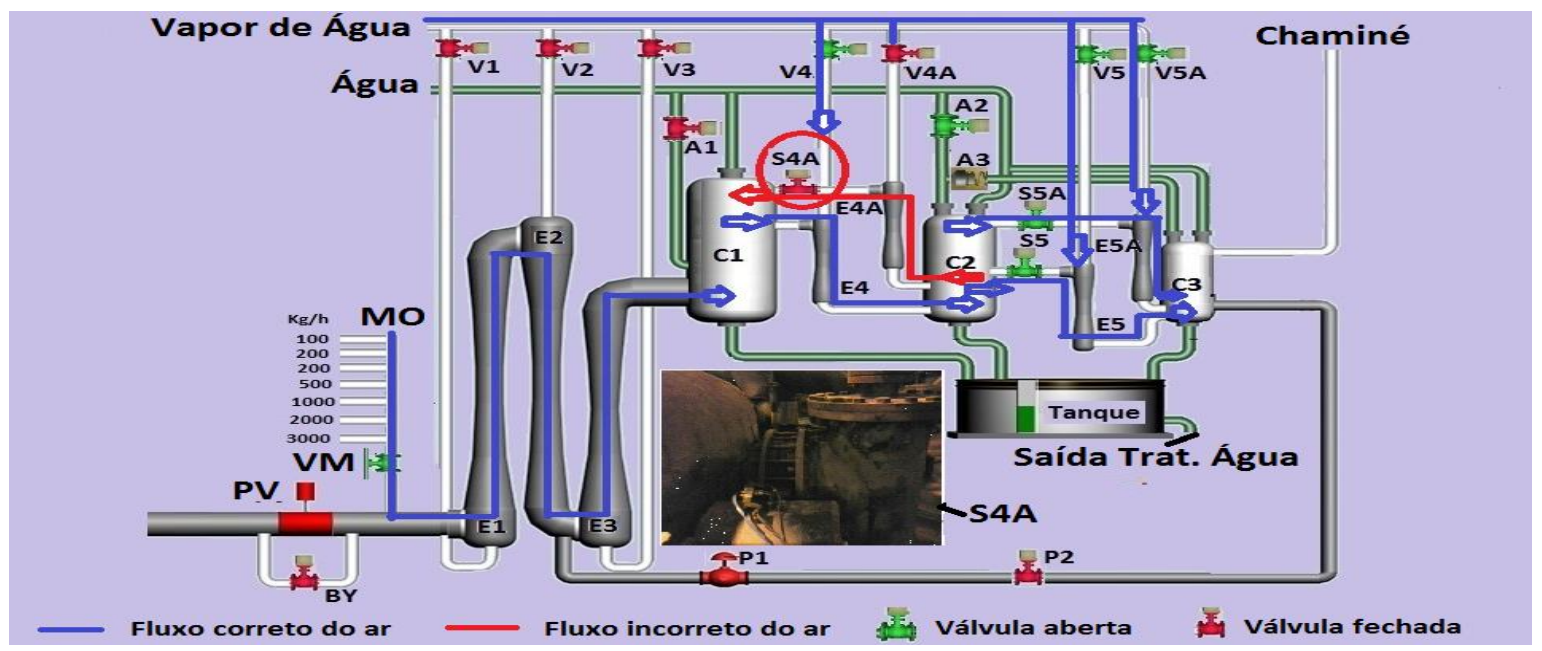

Figura 5: Recirculação através de S4A.

Abrem-se as válvulas shut off S5A e S5 da linha de sucção e depois as válvulas V5A, V5 e V4 da linha de vapor, todas através do supervisório. Dessa forma, os três ejetores E4, E5 e E5A estão funcionando. Abre-se também a válvula A2, que libera água para os condensadores $\mathrm{C} 2$ e C3.

Então, com P2 já testada e A2 aberta, consequentemente A3 está com passagem plena de água, não sendo possível existir um retorno de gás e vapor por ela de C2 para $\mathrm{C1}$. A válvula V5A também aberta, não sendo possível um retorno de gás e vapor por S5A, de C3 para C2, pois o E5A está funcionando e forçando gás e vapor para baixo. Só poderá existir passagem interna se uma válvula permitir passagem de fluxo no momento que ela está fechada. A passagem interna em A3 e S5A será detalhada nos experimentos à frente.

Para cada um dos experimentos a seguir, para testar a válvula S4A, aguardou-se por volta de 5 minutos, que foi o tempo necessário para a bomba de vácuo se estabilizar em uma determinada pressão. A pressão de vácuo atingida nos experimentos realizados foi de $4,72 \mathrm{kPa}$ (47,2 mbar) e a variação encontrada na pressão de vácuo foi de $\pm 0,04 \mathrm{kPa}$. Encerrado o vácuo, inundou-se com ar atmosférico a bomba de vácuo e aguardou-se a pressão da bomba se estabilizar. Aberta a válvula manual VM do manifold e as tampas para permitir um fluxo de ar de $800 \mathrm{~kg} / \mathrm{h}$. Repetido o experimento, o valor encontrado foi de 6,09 kPa (60,9 mbar) e, para $2.000 \mathrm{~kg} / \mathrm{h}$, o valor encontrado foi de $7,87 \mathrm{kPa}(78,7 \mathrm{mbar})$.

Para determinar quais seriam os valores de pressão de vácuo se a válvula S4A estivesse com passagem interna plena, aciona-se a sua abertura através do supervisório, sem abrir V4A. Após aberta S4A, é criada uma passagem livre de C2 para C1. Repetidos os experimentos já citados, encontra-se o valor de $6,5 \mathrm{kPa}$ (65 mbar) com a válvula VM fechada, e de 10,58 kPa (105,8 mbar) para uma entrada de ar de $800 \mathrm{~kg} / \mathrm{h}$ de ar e de 15,26 kPa (152,6 mbar) com $2000 \mathrm{~kg} / \mathrm{ar}$ através do manifold. $\mathrm{Na}$ simulação de passagem interna por $\mathrm{S} 4 \mathrm{~A}$, a variação de pressão é de $\pm 0,09 \mathrm{kPa}(0,9 \mathrm{mbar})$. O tempo para estabilizar a pressão de vácuo sem a abertura de S4A foi de 5 minutos. A tabela III contém os valores de referência obtidos. 
Tabela III Valores de pressão do sistema com e sem falha em S4A

\begin{tabular}{|c|c|c|}
\hline $\begin{array}{c}\text { Vazão de Ar } \\
\text { pelo Manifold }\end{array}$ & $\begin{array}{c}\text { Pressão de Vácuo } \\
\text { sem passagem interna em } \\
\text { S4A }\end{array}$ & $\begin{array}{r}\text { Pressão de vácuo com } \\
\text { passagem interna em S4A }\end{array}$ \\
\hline $0 \mathrm{~kg} / \mathrm{h}$ & $4,72 \mathrm{kPa}(47,2 \mathrm{mbar})$ & $6,50 \mathrm{kPa}(65 \mathrm{mbar})$ \\
\hline $800 \mathrm{~kg} / \mathrm{h}$ & $6,09 \mathrm{kPa}(60,9 \mathrm{mbar})$ & $10,58 \mathrm{kPa}(105,8 \mathrm{mbar})$ \\
\hline $2000 \mathrm{~kg} / \mathrm{h}$ & $7,87 \mathrm{kPa}(78,7 \mathrm{mbar})$ & $15,26 \mathrm{kPa}(152,6 \mathrm{mbar})$ \\
\hline
\end{tabular}

Para corrigir a falha até que S4A seja trocada deve-se forçar a abertura de V4A. A passagem de vapor por E4A não permite o retorno de gás e vapor para $C 1$ através de S4A, evitando a recirculação. A consequência é o aumento de temperatura em $\mathrm{C} 2$, pois no momento de abertura de $\mathrm{V} 3$, em $30,0 \mathrm{kPa}$ (300 mbar), a água que vai para $\mathrm{C} 2$ e C3 é retida pela válvula $A 2$ e essa água vai para $\mathrm{C} 1$ através da abertura automática de A1 junto com V3. A bomba de vácuo fica menos eficiente e há perda energética através do vapor em E4A, mas evita-se recirculação interna.

\subsection{VÁLVULA SHUT OFF DE PASSAGEM GÁS S5A}

Durante o tratamento de corridas, E5A,V5A e S5A são abertas apenas no início do processo. Por isso, a linha de sucção entre C2 e C3, passando por E5A, é paralela à linha de sucção entre C2 e C3, passando por E5. Perceba que existe a válvula shut off S5 na linha de sucção entre C2 e C3, passando por E5, mas ela nunca é fechada durante o tratamento de corridas nem durante este teste. A válvula S5 somente é fechada nos testes de infiltração externa da bomba de vácuo e teste de infiltração externa do sistema completo, não abordados neste artigo.

Como P2 e S4A já foram testadas, A2 está aberta e consequentemente A3 está com passagem plena de água, não sendo possível existir um retorno de gás e vapor por ela de C2 para C1. A V4A também está aberta não sendo possível um retorno de gás e vapor por S4A de C2 para C1 pois o E4A está funcionando e forçando gás e vapor para baixo. Os procedimentos para encontrar as referências de S5A são equivalentes para S4A. O de estabilização da pressão de vácuo, sem a abertura de S5A, é de 8 minutos. Na figura 6 é possível verificar o caminho da passagem interna por S5A.

Realiza-se para a válvula $S 5 A$ testes equivalentes aos realizados para S4A, considerando a bomba de vácuo em perfeito funcionamento, além dos valores de referência com a válvula shut off S5A em falha completa. A variação de pressão de estabilidade foi de $\pm 0,05 \mathrm{kPa}(0,5 \mathrm{mbar})$ sem passagem interna pela válvula e de \pm $0,06 \mathrm{kPa}(0,6 \mathrm{mbar})$ com passagem interna pela válvula. 


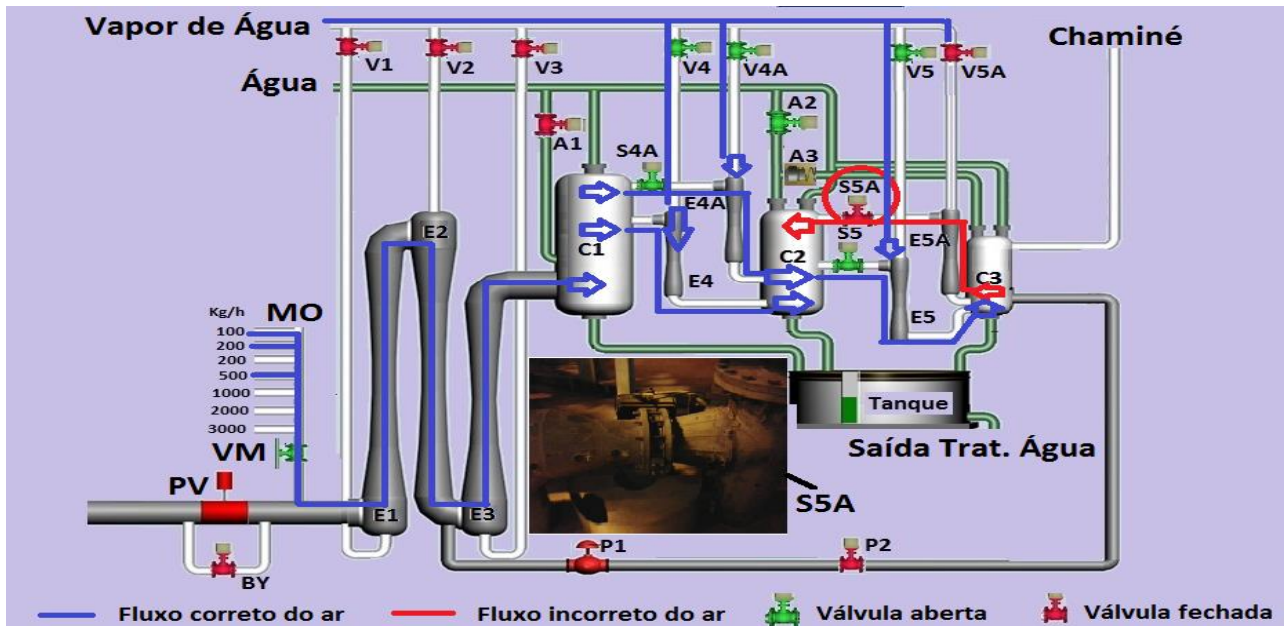

Figura 6: Recirculação através de S5A.

Tabela IV Valores de pressão do sistema com e sem falha em S5A.

\begin{tabular}{|c|c|c|}
\hline $\begin{array}{c}\text { Vazão de Ar } \\
\text { pelo Manifold }\end{array}$ & $\begin{array}{c}\text { Pressão de vácuo sem } \\
\text { passagem interna em S5A }\end{array}$ & $\begin{array}{c}\text { Pressão de vácuo com } \\
\text { passagem interna plena em S5A }\end{array}$ \\
\hline $0 \mathrm{~kg} / \mathrm{h}$ & $4,35 \mathrm{kPa}(43,5 \mathrm{mbar})$ & $69,40 \mathrm{kPa}(694 \mathrm{mbar})$ \\
\hline $800 \mathrm{~kg} / \mathrm{h}$ & $5,06 \mathrm{kPa}(50,6 \mathrm{mbar})$ & $70,20 \mathrm{kPa}(702 \mathrm{mbar})$ \\
\hline $2000 \mathrm{~kg} / \mathrm{h}$ & $8,90 \mathrm{kPa}(89,0 \mathrm{mbar})$ & $70,65 \mathrm{kPa}(706,5 \mathrm{mbar})$ \\
\hline
\end{tabular}

$\mathrm{Na}$ condição de tratamento de uma corrida (simulação com $800 \mathrm{~kg} / \mathrm{h}$ de ar), caso a válvula S4A apresente passagem interna plena, a bomba de vácuo se estabiliza em $10,58 \mathrm{kPa}$ e se a ocorrência for com a S5A, a bomba de vácuo se estabiliza com $70,2 \mathrm{kPa}$. As duas condições anteriores valem para o momento em que o ejetor E3 ainda não entrou em funcionamento.

A forma de corrigir a falha até que S5A seja trocada, é simplesmente forçar a abertura de V5A. A passagem de vapor por E5A não irá permitir o retorno de gás $\mathrm{e}$ vapor para C2 através de S5A, evitando a recirculação. A consequência será um aumento de temperatura em $\mathrm{C} 3$, pois no momento de abertura de $\mathrm{V} 3$, em $30 \mathrm{kPa}$, a água que vai para C2 e C3 será fechada através da válvula A2 e essa água irá para C1 através da abertura automática de A1 junto com V3. No geral, a bomba de vácuo será menos eficiente e haverá perda de energia através do vapor por E5A, que não terá função de reduzir a pressão de vácuo, mas evitar a recirculação interna.

\subsection{VÁLVULA A3 DE RETENÇÃO DE ÁGUA DOS CONDENSADORES}

Os testes de recirculação a serem realizados na válvula $A 3$ devem ser acompanhados juntamente com a figura 7 . 


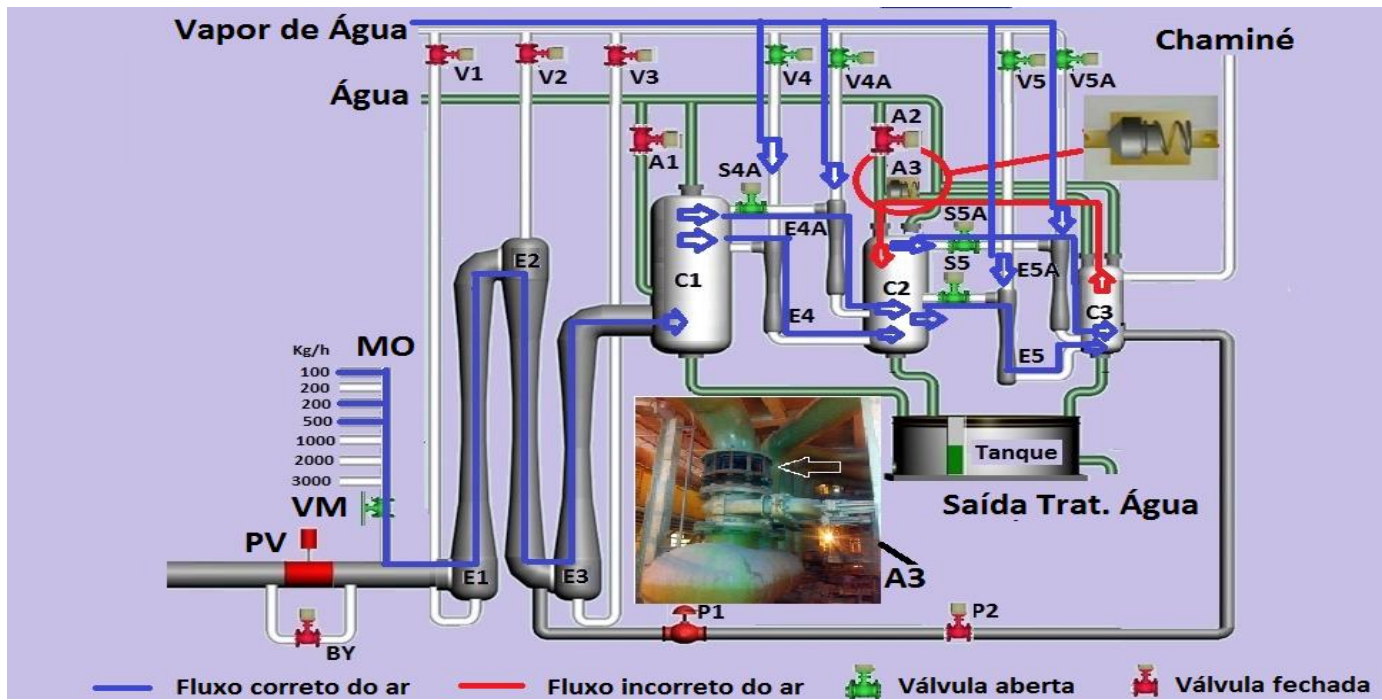

Figura 7: Recirculação através de A3.

Durante o tratamento de corridas, no momento que o sistema atinge $10 \mathrm{kPa}$, a válvula A2 fecha e A1 abre. Essa troca de água de $\mathrm{C} 2$ e $\mathrm{C} 3$ para $\mathrm{C} 1$ é devido ao fechamento das válvulas V4A e V5A, que trabalham como motor de arranque até o sistema atingir essa pressão para $\mathrm{V} 3$ abrir. Com menor vazão de vapor em C2 e C3 e maior vazão em C1, a vazão de agua é comutada. Como a tubulação que passa por A3 está sem água, pode existir passagem de gás e vapor por ela em caso de falha, existindo a passagem interna (vide figura 7).

Os procedimentos dos experimentos para a válvula de retenção $A 3$ são os mesmos para P2 já realizados, com exceção da não abertura da válvula A2. Consequentemente, A3 permanece fechada para não existir fluxo de água por ela, tornando possível a identificação de passagem interna de C2 para C1. Os valores de referência estão na tabela $\mathrm{V}$. O tempo para este teste deve ser curto, devido à vazão de vapor em C2 e C3 com somente a metade da água necessária pois a válvula A2 estava fechada. A variação de temperatura em C3 foi de $343 \mathrm{~K}\left(70^{\circ} \mathrm{C}\right)$, em C2 foi a $323 \mathrm{~K}\left(50^{\circ} \mathrm{C}\right)$, muito maiores que os $291 \mathrm{~K}\left(18^{\circ} \mathrm{C}\right)$ característicos.

Tabela V: Valores de pressão do sistema sem falha em A3.

\begin{tabular}{|c|r|c|}
\hline $\begin{array}{c}\text { Vazão de Ar } \\
\text { pelo Manifold }\end{array}$ & $\begin{array}{c}\text { Pressão de vácuo sem } \\
\text { passagem interna em A3 }\end{array}$ & $\begin{array}{c}\text { Pressão de vácuo com } \\
\text { passagem interna plena em A3 }\end{array}$ \\
\hline $0 \mathrm{~kg} / \mathrm{h}$ & $4,00 \mathrm{kPa}(40,0 \mathrm{mbar})$ & $>4,00 \mathrm{kPa}(40,0 \mathrm{mbar})$ \\
\hline $800 \mathrm{~kg} / \mathrm{h}$ & $4,99 \mathrm{kPa}(49,9 \mathrm{mbar})$ & $>4,99 \mathrm{kPa}(49,9 \mathrm{mbar})$ \\
\hline $2000 \mathrm{~kg} / \mathrm{h}$ & $5,75 \mathrm{kPa}(57,5 \mathrm{mbar})$ & $>5,75 \mathrm{kPa}(57,5 \mathrm{mbar})$ \\
\hline
\end{tabular}

Existem duas formas de impedir a circulação interna de gás e vapor por $A 3$ até que ela seja trocada. A primeira delas é acionando a abertura de A2 através do supervisório, porque o fluxo de água impede o retorno de vapor e gás. Consequentemente, reduz-se a vazão de água em $\mathrm{C} 1$ quando o sistema está abaixo de 10,0 kPa (100 mbar). A segunda forma é fechar uma válvula manual que existe logo antes de A3. Esta válvula não está em nenhuma das figuras anteriores. A função desta válvula manual na linha de água é facilitar a troca de A3, durante uma manutenção, fechando o fluxo. A consequência deste procedimento é a restrição de água em $\mathrm{C} 2$ e $\mathrm{C} 3$ e aumento de sua temperatura interna no início do processo, quando E5 e E5A estão em funcionamento, até o sistema atingir 10,00 kPa (100 
mbar). Ambos os procedimentos reduzem a eficiência da bomba de vácuo, mas ainda são melhores do que permitir a passagem interna em A3.

\section{CONCLUSÃO}

Conforme Oliva[1], novos sistemas de geração de vácuo com bombas a seco tenderão a serem mais utilizados; entretanto, plantas com ejetores a vapor ainda dominam o mercado siderúrgico, além de plantas de unidade crua de petróleo, conforme Martin[4]. Conhecer a fundo o processo de formação de vácuo através do vapor, a circulação dos gases, os diferentes estágios de pressão e os modos de falha são primordiais à obtenção de valores de referência. A partir destes experimentos, testes foram realizados para comparação de dados e conclusões sobre o sistema.

Os experimentos aqui descritos possuem procedimentos lógicos, fruto de experiências de diversos tratamentos de falha, estudo sobre o processo e equipamentos. Cada processo possui seus parâmetros de vapor, água, dimensões de equipamentos, layout de válvulas, ejetores e condensadores, mas nada impede que outros profissionais inspirados neste artigo estudem seus processos e desenvolvam outros testes e procedimentos. Este estudo conseguiu atingir os seguintes resultados:

- Encontrar os valores de referência para o sistema de vácuo existente em planta.

- Realizar testes de rotina e compará-los aos valores de referência obtidos.

- Identificar a falha responsável pela recirculação de gases no sistema.

- Reduzir o tempo de detecção e correção das falhas.

- Reduzir perdas de produção, porque intervenções serão planejadas com a devida antecedência.

- Aperfeiçoar a política de sobressalentes, conhecendo quais componentes têm maior influência na recirculação de gases e vapor.

\section{Agradecimentos}

Agradeço a Gerdau Ouro Branco pela possibilidade de realizar o estudo, experimentos e apresentação no Congresso Anual da ABM.

\section{REFERÊNCIAS}

1 OLIVA, V.R. Análise de Viabilidade Técnica-Econômica na Substituição de um Sistema Combinado de Geração de Vapor-Vácuo por Bombas Secas. Universidade Estadual Paulista, Araraquara, 2013. 
2 DEPARTMENT OF AEROSPACE AND OCEAN ENGINEERING, VIRGINIA TECH. Nozzle Applet. Disponível em <www.engapplets.vt.edu/fluids/CDnozzle/cdinfo.html >. Acesso em 05 de agosto de 2015.

3 EL-DESSOUKY H. et al. Evaluation of Stem Jet Ejectors. College of Engineering and Petroleum, Kuwait University, 2002

4 MARTIN, G. Understand Real World Problems of Vacuum Ejector Performance.

Process Consulting Services Inc., Houston, Texas, 1997 